\title{
Editorial
}

\section{Medication-related osteonecrosis of jaws revisited through the bone inherited disorders: what do we know?}

\author{
Mathilde Fénelon ${ }^{1,2, *}$, Jean-Christophe Fricain ${ }^{1,2}$ \\ ${ }^{1}$ Service de chirurgie orale, CHU de Bordeaux, France \\ ${ }^{2}$ Centre de compétence des maladies rares orales et dentaires (0-Rares), CHU de Bordeaux, France
}

(Received: 29 April 2021, accepted: 29 April 2021)

Today, oral surgeons routinely meet bone complications in patients receiving anti-resorptive treatments. Bisphosphonates and Denosumab are both osteoclast-targeted anti-resorptive therapy which may cause osteonecrosis of the jaw, also known as Medication-Related Osteonecrosis of Jaws (MRONJ) [1]. However, such osteonecrosis of the jaw are not a new phenomenon since they are reminiscent of earlier pathologies which comparable bone manifestations. This type of jaw osteonecrosis was already reported after exposure to white phosphorus or radium. Furthermore, osteonecrosis of the jaw might also be caused by hereditary bone disorders. Osteopetrosis and pycnodysostosis are rare, heritable disorders of the skeleton characterized by increased bone density [2]. They can sometimes lead to maxillofacial complications, such as osteomyelitis of the jaw, whose symptomatology is very similar to that of MRONJ. We believed that studying this history of osteomyelitis associated with hereditary bone disorders might provide some relevant information to the current situation with bisphosphonates.

\section{Ostenonecrosis underlying mechanism}

Bisphosphonates inhibit bone resorption by inducing apoptosis of mature osteoclasts, and denosumab acts by preventing the formation of osteoclasts from their precursors. Osteopetrosis refers to a group of rare, inherited bone diseases, characterized by increased bone density due to impaired bone resorption by osteoclasts and, more specifically, to an abnormality in osteoclast development or function. Pycnodysostosis (PYCD) is a rare osteosclerotic dysplasia occurring from a mutation in the gene that encodes cathepsin K, an enzyme secreted by osteoclasts that is responsible for the degradation of bone matrix protein. Cathepsin K deficiency affects bone remodeling, leading to osteosclerosis.

\footnotetext{
*Correspondence: mathildefenelon@live.fr
}

The deficit of bone resorption by inhibition of osteoclastic activity is therefore the common pathophysiological mechanism of these hereditary osteosclerotic diseases and antiresorptive drugs, which explains the similarity of the bone complications found in these patients.

\section{Clinical and radiological manifestations}

While the term "osteonecrosis" is used when the bone damage is related to antiresorptive drugs, "osteomyelitis" is used when it occurs in the course of an inherited bone disease. MRONJ refers to necrotic bone, whereas the term "osteomyelitis" implies a notion of diffuse infection of the bone and bone marrow. However, their clinical manifestations are very similar such as: exposed and necrotic bone or fistulae with or without evidence of infection. Moreover, it is difficult to clearly differentiate primary osteomyelitis from primary osteonecrosis of the jaw, as the proximity and abundance of oral bacterial flora might cause a secondary infection and complicates MRONJ.

In the initial stage, most of these osteitis are not accompanied by radiological signs, the radiological manifestations often being delayed in relation to the clinic. Increased bone density (osteosclerosis) can then be observed radiologically, as well as thickening of the lamina dura, non-healing of extraction sockets and limited or extended osteolysis. In late stage, pathologic fracture can also be observed.

\section{Occurrence}

MRONJ and osteomyeilitis in osteopetrosis and pycnodysostosis seem to be located exclusively in jaws. The mandible shows a higher incidence. Regardless of the etiology, osteonecrosis occur either spontaneously or a precipitating event that produced the bone exposures can be identified. Bacterial contamination following dental infection is often reported. 


\section{Treatment modalities}

Strategies in the treatment also share similarities. They depend on the localization and size/extension of the dead bone. The first-line of treatment is conservative with antibiotics, associated or not with curettage, sequestrectomy or decortication. 0therwise, interrupting bone resection and reconstruction by plate or free flap has also been reported for more advanced forms. Several authors have reported difficulties in obtaining mucosal healing and bone consolidation in these patients.

Osteonecrosis related to anti-resorptive treatments is a form of drug-induced osteosclerosis, with a similar clinical course to that of genetically related diseases (i.e. osteopetrosis and pycnodysostosis). Finally, regardless of the etiology, jaw osteonecrosis is the ultimate consequence of loss of bone remodeling and renewal through impaired osteoclastic function.

\section{References}

1. Voss PJ, Poxleitner P, Schmelzeisen R, Stricker A, Semper-Hogg W. Update MRONJ and perspectives of its treatment. J Stomatol Oral Maxillofac Surg 2017;118:232-235.

2. Fénelon $M$, Catros $S$, Boisramé $S$, $D^{\prime}$ incau $E$, Tréguer $A$, Gobel $Y$, Valette G, Fricain JC. Complications maxillo-faciales associées à la pycnodysostose: présentation de deux cas cliniques et revue de la littérature. Méd Buccale Chir Buccale 2015;21:169-176. 